\title{
Applications of the CRISPR/Cas9 genome editing system for modification of starch content in wheat and triticale
}

\author{
Timerbaev V. ${ }^{1,2,3 *}$, Miroshnichenko D. ${ }^{1,2,3}$, Bazhenov M. ${ }^{1}$, Klementyeva A. ${ }^{2,3}$, \\ Bespalova L.. ${ }^{4}$ Divashuk M.G. ${ }^{1,3}$, Karlov G. ${ }^{3}$, Kroupin P. ${ }^{1,3}$, Dolgov S..$^{2,3}$ \\ ${ }^{1}$ Kurchatov Genomic Center - ARRIAB, All-Russia Research Institute of Agricultural Biotechnology, \\ Moscow, Russia \\ ${ }^{2}$ Shemyakin and Ovchinnikov Institute of Bioorganic Chemistry RAS, Pushchino, Russia \\ ${ }^{3}$ All-Russia Research Institute of Agricultural Biotechnology, Moscow, Russia \\ ${ }^{4}$ Department of Breeding and Seed Production of Wheat and Triticale, National center of grain named \\ after Lukyanenko, Central Estate of KNIISH, Krasnodar, Russia \\ *email:timerbaev@gmail.com
}

Starch is the major component of mature cereal grains, representing more than $65 \%$ of its dried weight. The capacity for starch synthesis during grain filling can influence final grain weight, while the starch composition is an important factor for the technological and nutritional properties of flours. There is an increasing need to develop cereal crops with higher amylose content to improve human health and lower the risk of serious noninfectious diseases. Recent advances in technologies for genetic transformation and genome editing have opened up new perspectives for precise starch manipulation in cereals. In the present study, a CRISPR/Cas9 approach was used to modify the starch accumulation in two important cereal crops, such as emmer wheat (Triticum dicoccum) and triticale $(\times$ Triticosecale). Using a modular cloning system, two expression cassettes are designed to simultaneously knockout several genes involved in the grain starch composition and properties. Each polycistronic construct consists of six various corresponding sgRNAs under the control of the TaU3 wheat promoter to induce simultaneous indel mutation in such genes as the granule bound starch synthase (GBSSI), the starch branching enzyme (SBEIIa), the starch synthase (SSIIa), starch debranching enzymes of isoamylase (ISAI) and the starch regulator 1 (RSR1). Using a co-transformation strategy, polycistronic constructs were delivered by gene gun into morphogenic cereal cells together with an expression vector encoding the Cas 9 nuclease to achieve the mutagenesis. The generation of genome-edited plants demonstrating the targeted modifications of native genes will be discussed.

Funding: Kurchatov Genomic Center of All-Russia Research Institute of Agricultural Biotechnology, agreement No. 075-15-2019-1667. 